\title{
REVIEW
}

\section{The development of evidence-informed physical activity guidelines for adults with spinal cord injury}

\author{
KA Martin Ginis ${ }^{1}$, AL Hicks ${ }^{1}$, AE Latimer ${ }^{2}$, DER Warburton ${ }^{3}$, C Bourne ${ }^{4}$, DS Ditor ${ }^{5}$, DL Goodwin ${ }^{6}$, \\ KC Hayes $^{7}$, N McCartney ${ }^{1}$, A Mcllraith ${ }^{8}$, P Pomerleau ${ }^{9}, \mathrm{~K} \mathrm{Smith}^{10}$, JA Stone $^{11}$ and DL Wolfe \\ ${ }^{1}$ Department of Kinesiology, McMaster University, Hamilton, Ontario, Canada; ${ }^{2}$ School of Kinesiology and Health Studies, Queen's \\ University, Kingston, Ontario, Canada; ${ }^{3}$ Experimental Medicine Program, Faculty of Medicine, University of British Columbia, \\ Vancouver, British Columbia, Canada; ${ }^{4}$ Active Living Alliance for Canadians with a Disability, Ottawa, Ontario, Canada; \\ ${ }^{5}$ Department of Kinesiology, Brock University, St Catharines, Ontario, Canada; ${ }^{6}$ Faculty of Physical Education and Recreation, \\ The Steadward Centre for Personal \& Physical Achievement, University of Alberta, Edmonton, Alberta, Canada; ${ }^{7}$ Department of \\ Physical Medicine and Rehabilitation, University of Western Ontario, London, Ontario, Canada; ${ }^{8}$ Consumer (No Affiliation); \\ ${ }^{9}$ Institut de Réadaptation en Déficience Physique du Québec, Quebec City, Quebec, Canada; ${ }^{10}$ Department of Physical Medicine \\ \& Rehabilitation, St Mary's of the Lake Hospital, Kingston, Ontario, Canada and ${ }^{11}$ Faculty of Medicine, University of Calgary, \\ Calgary, Alberta, Canada
}

\begin{abstract}
Objectives: To systematically develop evidence-informed physical activity guidelines to improve physical fitness in people with spinal cord injury $(\mathrm{SCl})$.

Setting: This study was conducted in Canada

Methods: The Appraisal of Guidelines, Research and Evaluation II guideline development protocol was used to develop exercise guidelines to improve physical capacity and muscular strength. The evidence base for the guideline development process consisted of a systematic review and quality appraisal of research examining the effects of exercise on physical fitness among people with $\mathrm{SCl}$. A multidisciplinary expert panel deliberated the evidence and generated the guidelines. Pilot testing led to refinement of the wording and presentation of the guidelines.

Results: The expert panel generated the following guidelines: for important fitness benefits, adults with a SCl should engage in (a) at least 20 min of moderate to vigorous intensity aerobic activity two times per week and (b) strength training exercises two times per week, consisting of three sets of 8-10 repetitions of each exercise for each major muscle group.

Conclusion: People with $\mathrm{SCl}$, clinicians, researchers and fitness programmers are encouraged to adopt these rigorously developed guidelines.

Spinal Cord (2011) 49, 1088-1096; doi:10.1038/sc.2011.63; published online 7 June 2011
\end{abstract}

Keywords: exercise; strength training; aerobic training; clinical practice guidelines; spinal cord injuries

\section{Introduction}

The amount of disability associated with a spinal cord injury (SCI) is unique to each injury and depends on both the level and completeness of damage to the spinal cord. However, physical deconditioning is a common consequence of most SCIs, and can further exacerbate the impact of the injury and lead to an increased risk for chronic secondary health complications. It has been suggested that much of the excessive (and early) morbidity and mortality in people with chronic SCI is caused by inactivity related illnesses, such as cardiovascular disease, type II diabetes and osteoporosis. ${ }^{1}$ In

Correspondence: Dr KAM Ginis, Department of Kinesiology, McMaster University, Hamilton, Ontario, Canada L8S 4K1.

E-mail: martink@mcmaster.ca

Received 22 February 2011; revised 26 April 2011; accepted 4 May 2011; published online 7 June 2011 addition, the loss of fitness and independence associated with physical inactivity significantly impacts quality of life and community participation. ${ }^{2}$ These facts highlight the importance of promoting physical activity (PA) to improve health, fitness and overall quality of life within the SCI population. ${ }^{3,4}$

PA promotion is very difficult in the absence of information regarding the types, amounts and intensities of activity that yield fitness benefits. Such information would assist clinicians and exercise programmers in prescribing and promoting exercise and PA. The able-bodied population has access to this type of information in the form of PA guidelines. $^{5-7}$ However, the able-bodied PA guidelines are not necessarily appropriate for people with SCI. The rigorous development of evidence-based PA guidelines that are specific to the needs and capabilities of the SCI population is long overdue. ${ }^{8,9}$ This paper describes the methodological 
approach underlying the development of PA guidelines to improve fitness in people with SCI.

It should be noted that other authors have published exercise recommendations for the SCI population. ${ }^{10-12}$ However, these recommendations have not been underpinned by a robust, standardized guideline development process that combines rigorous methodology (for example, a systematic review of research evidence, pilot testing) with the meaningful engagement of a multidisciplinary team of stakeholders to deliberate the research evidence and generate consensus guidelines. For example, the recommendations of Jacobs and $\mathrm{Nash}^{11}$ were based on a narrative review (as opposed to a systematic review) that did not take into account the quality of the evidence or the perspectives of key stakeholders. The ACSM ${ }^{10}$ guidelines provide general training parameters for beginner and experienced exercisers with 'spinal cord dysfunction,' but these parameters are not linked to a supporting body of literature. The ACSM also provides a guideline of 'exercise every week for at least three sessions per week', ${ }^{10}$ but does not provide an evidence base to indicate whether this prescription represents a threshold for achieving fitness or other health benefits. Given these shortcomings, the purpose of the present project was to develop evidence-informed, consensus PA guidelines to improve fitness among people with SCI.

We harmonized our guideline development process with that of the Canadian Society for Exercise Physiology (CSEP). From 2006 to 2010, CSEP undertook the process of revising the Canadian PA guidelines for four groups: adults, older adults, children and adolescents. CSEP implemented steps to ensure rigor, comprehensiveness and transparency in the guideline development process by adopting the internationally recognized Appraisal of Guidelines, Research and Evaluation (AGREE II) ${ }^{13-15}$ protocol along with a strategy for assessing the quality of research evidence used to formulate the guidelines. ${ }^{9}$ To the best of our knowledge, this paper describes the first attempt to develop SCI-specific PA guidelines using this same rigorous, cutting edge protocol for guideline development in conjunction with a quality assessment and grading of the research evidence. In the following sections, we describe this process and the resulting PA guidelines. For brevity, this information has been merged into a combined Methods and Results section.

\section{Methods and results}

\section{Overview}

The 23-item AGREE II instrument ${ }^{13-15}$ was used as a framework for developing the guidelines. The 23 items represent six domains that capture key reporting criteria for a practice guideline; (1) scope and purpose: the overall objectives of the guideline, the specific clinical questions addressed by the guideline and the target population; (2) stakeholder involvement: the extent to which the guideline represents the perspectives of the end-user groups; (3) rigor of development: the process used to gather and synthesize evidence, to make recommendations for guideline content and to update the guideline regularly; (4) clarity and presentation: the language, format and implementation tools used to communicate the guideline; (5) applicability: the anticipated implications and costs of implementing the guideline; and (6) editorial independence: the acknowledgement of potential biases because of the project funding or conflicts of interest among individuals who participated in the guideline development process.

We used AGREE II as a step-by-step guide to the development process. Briefly, the project leaders (KMG, AH) determined the general scope and purpose of the project and conducted a systematic review of relevant literature. ${ }^{16}$ A consensus panel of stakeholders was convened to refine the scope and purpose, to review the evidence, to make recommendations for the guideline content and to consider the implications for implementing and updating the guidelines. A draft of the guidelines was prepared and disseminated to additional stakeholders for feedback. Once the guidelines and this manuscript (describing the guideline development) were prepared, an independent methodological consultant with training in the AGREE II process reviewed the materials and evaluated them using AGREE II (report available from the first author).

\section{Guideline development process}

This section outlines the process in terms of the six AGREE II quality domains.

Guideline scope and purpose. The project leaders (KMG, AH) determined the general objectives, clinical questions and target population.

- Objective: To create guidelines to increase physical fitness among people with SCI.

- Clinical questions: What is the minimum frequency, intensity, duration and type of training needed to generate fitness benefits among people with SCI?

- Target population: Adults with traumatic or non-traumatic SCI (ages 18-64), including tetraplegia and paraplegia.

During the evidence review process, the consensus panel refined the objectives, clinical questions and target population (see 'rigor of development').

Stakeholder involvement. The project leaders (KMG, AH) have expertise in PA for people with SCI. They led a subgroup in conducting a systematic review of research examining the effects of exercise training on physical fitness among people with SCI. ${ }^{16}$ In all, 12 additional people from across Canada comprised the consensus panel. The panel included experts in the AGREE II process (JS) and with experience creating clinical practice guideline (AH, JS, NM, KMG, AL and DERW). Panel members included researchers from multiple disciplines: knowledge translation (KH, DW), exercise behavior change (KMG, $\mathrm{AL}$ ) and exercise physiology (AH, DD, NM, KH, DW). As well, the panel included endusers representing various interest groups: people with SCI (AM, CB), qualified exercise professionals (that is, CSEPCertified Exercise Physiologists and CSEP-Certified Personal Trainers (DERW), SCI Physical Education Specialist (PP), 
Table 1 Expert panel

\begin{tabular}{|c|c|c|}
\hline \multicolumn{3}{|c|}{ Expert panel } \\
\hline Name & Expertise and institution & Role(s) \\
\hline Dr Kathleen Martin Ginis (PhD) & $\mathrm{SCl}$, health and exercise psychology & Co-principal investigator \\
\hline Dr Audrey Hicks (PhD) & $\begin{array}{l}\text { Researcher: McMaster University } \\
\text { SCI, neuromuscular physiology and exercise rehabilitation } \\
\text { Researcher: McMaster University }\end{array}$ & $\begin{array}{l}\text { Content expert, Systematic review, writing team } \\
\text { Co-principal investigator } \\
\text { Content expert, systematic review, writing team }\end{array}$ \\
\hline Dr James Stone (MD) & $\begin{array}{l}\text { Exercise and cardiovascular physiology } \\
\text { University of Calgary/Cardiac Wellness Institute of Calgary }\end{array}$ & Chair of expert panel \\
\hline Chris Bourne (MA) & $\begin{array}{l}\mathrm{SCl} \text { consumer; physical activity and disability } \\
\text { Community Development manager: Active Living Alliance }\end{array}$ & Content expert \\
\hline Dr Dave Ditor (PhD) & $\begin{array}{l}\mathrm{SCl} \text {, exercise physiology } \\
\text { Researcher: Brock University }\end{array}$ & Content expert \\
\hline Dr Donna Goodwin (PhD) & $\begin{array}{l}\mathrm{SCl} \text {, exercise community programming } \\
\text { Director: Steadward Centre, Edmonton }\end{array}$ & Content expert \\
\hline Dr Keith Hayes (PhD) & $\begin{array}{l}\mathrm{SCl} \text {, exercise physiology knowledge translation } \\
\text { Researcher: University of Western Ontario }\end{array}$ & Content expert \\
\hline Dr Amy Latimer (PhD) & $\begin{array}{l}\mathrm{SCl} \text {, exercise behavior change } \\
\text { Researcher: Queen's University }\end{array}$ & Content expert, writing team, pilot testing \\
\hline Dr Neil McCartney (PhD) & $\begin{array}{l}\mathrm{SCl} \text {, exercise rehabilitation } \\
\text { Researcher: McMaster University }\end{array}$ & Content expert \\
\hline Audrey Mcllraith (MSc) & $\mathrm{SCl}$ consumer & Content expert \\
\hline Pierre Pomerleau (MSc) & $\begin{array}{l}\mathrm{SCl} \text { physical education specialist } \\
\text { Quebec IRDPQ }\end{array}$ & Content expert \\
\hline Dr Karen Smith (MD) & $\begin{array}{l}\mathrm{SCl} \text {, physiatry } \\
\text { Clinician and researcher: Queen's University }\end{array}$ & Content expert \\
\hline Dr Darren ER Warburton (PhD) & $\begin{array}{l}\mathrm{SCl} \text {, exercise physiology guideline development } \\
\text { Researcher: University of British Columbia }\end{array}$ & Content expert, writing team \\
\hline Dr Dalton Wolfe (PhD) & $\begin{array}{l}\mathrm{SCl} \text {, knowledge translation, exercise physiology } \\
\text { Researcher: University of Western Ontario }\end{array}$ & Content expert, systematic review \\
\hline
\end{tabular}

Abbreviation: $\mathrm{SCl}$, spinal cord injury.

physicians (JS, KS) and service providers (DG, AH, AL, AM, $\mathrm{CB}, \mathrm{NM}$ ). Table 1 lists panel members, their expertise and roles. The panel did not include public policy makers or government officials.

The panel identified user groups as people with SCI, rehabilitation specialists, qualified exercise professionals, physicians and researchers. The resulting guidelines could be used to inform out-patient rehabilitation practice, provide a foundation for further PA research (for example, to explore the link between PA and disease) and to impact current standards of outpatient care.

During the panel meeting, the systematic review evidence $^{16}$ was thoroughly deliberated. Deliberations resulted in the production of a draft set of PA guidelines, together with clarification messages regarding possible activities to achieve the guidelines. Following the panel meeting, the draft guidelines were circulated to people with SCI and health care professionals for pilot testing and to give endusers an opportunity to express their views and preferences regarding the presentation of the guidelines. The university research ethics board approved this protocol.

The SCI sample $\left(N=16 ; 81 \%\right.$ male; $M_{\text {age }}=44.9$ years $\pm 11.8 ; M_{\text {years postinjury }}=18.8 \pm 14.2$ ) included both people with paraplegia (75\%) and tetraplegia (25\%). Most were manual $(56 \%)$ or power wheelchair users $(31 \%)$, and the remainder $(13 \%)$ walked independently or with a gait aid as their primary mode of mobility. The professional sample ( $N=44 ; 84 \%$ female) consisted of physiotherapists $(23 \%)$, occupational therapists (7\%), kinesiologists (21\%), physical therapists (27\%) and others (23\%) who worked with patients with SCI (86\% reported working 'all the time' or 'frequently' with this patient group). Roughly half (44\%) had prescribed PA to a person with SCI $<20$ times, $16 \%$ prescribed $20-40$ times and $41 \%$ had prescribed activity $>40$ times.

Participants completed a web-based or paper survey. On seven-point scales, they rated whether the guidelines were realistic, appropriate, feasible and useful, and whether the guidelines reflected the amount, type and intensity of PA that people with SCI like to do and are capable of doing. Higher scores corresponded with more favorable responses. It was predetermined that survey items with mean responses below the scale mid-point $(\leqslant 4)$ would indicate aspects of the guideline presentation requiring improvement. Participants were asked to explain their ratings and provide suggestions for improving the presentation of the guidelines.

Ratings for each survey item are presented in Table 2. Overall, respondents rated the guidelines positively. Only one item (Does the guideline provide practitioners with clear instructions about the intensity level?) had a mean rating $<4$. Given the quantity and quality of feedback received, the survey process was considered successful. Table 3 provides a summary of participants' feedback and how this information, coupled with the Table 2 results, was used to modify the presentation of the guidelines.

Rigor of development. A systematic review of research regarding exercise training and fitness outcomes in people with SCI provided the guideline evidence base. ${ }^{16} \mathrm{~A}$ full 
Table 2 Guideline ratings obtained from health care practitioners and individuals with $\mathrm{SCl}$

\begin{tabular}{|c|c|c|c|c|c|c|}
\hline \multirow[t]{2}{*}{ Content item } & \multicolumn{3}{|c|}{$\begin{array}{l}\text { Health care } \\
\text { practitioners }\end{array}$} & \multicolumn{3}{|c|}{$\begin{array}{l}\text { Individuals } \\
\text { with } \mathrm{SCl}\end{array}$} \\
\hline & $\mathrm{n}$ & $\mathrm{M}($ s.d.) & $\begin{array}{l}\text { Range of } \\
\text { responses }\end{array}$ & $\mathrm{n}$ & $\mathrm{M}($ s.d.) & $\begin{array}{l}\text { Range of } \\
\text { responses }\end{array}$ \\
\hline \multicolumn{7}{|l|}{ Perceived feasibility } \\
\hline Is the guideline appropriate for all individuals with $\mathrm{SCl}$ ? & 43 & $4.49(1.74)$ & $1-7$ & 16 & $5.19(1.64)$ & $2-7$ \\
\hline Is the guideline realistic if the person is motivated and has all resources necessary? & 44 & $5.78(1.27)$ & $3-7$ & 16 & $6.63(0.72)$ & $5-7$ \\
\hline $\begin{array}{l}\text { Does the guideline reflect the amount, type, and intensity of PA that people with } \\
\text { SCI like to do? }\end{array}$ & 44 & $4.70(1.44)$ & $1-6$ & 16 & $4.38(1.78)$ & $1-7$ \\
\hline How confident are you that you can meet this guideline?* & & & & 16 & $6.25(1.13)$ & $4-7$ \\
\hline How confident are you that people with paraplegia can meet this guideline?* & 43 & $5.23(1.46)$ & $1-7$ & & & \\
\hline How confident are you that people with tetraplegia can meet this guideline?* & 44 & $4.30(1.58)$ & $1-7$ & & & \\
\hline \multicolumn{7}{|l|}{ Perceived utility } \\
\hline Does the guideline provide useful information for people with $\mathrm{SCI}$ ? & 44 & $4.80(1.80)$ & $1-7$ & 16 & $5.50(1.63)$ & $2-7$ \\
\hline Does the guideline provide useful information for practitioners? & 44 & $4.45(1.77)$ & $2-7$ & & & \\
\hline Would you use this guideline in your practice? & 43 & $5.14(1.68)$ & $1-7$ & & & \\
\hline \multicolumn{7}{|l|}{ Presentation and clarity } \\
\hline \multicolumn{7}{|l|}{ Does the guideline } \\
\hline $\begin{array}{l}\text { provide practitioners/you clear instructions about how much PA should be } \\
\text { done in a week? }\end{array}$ & 43 & $4.77(1.63)$ & $2-7$ & 16 & $5.44(1.59)$ & $2-7$ \\
\hline provide practitioners/you clear instructions about the intensity level of PA? & 43 & $3.72(1.74)$ & $1-7$ & 16 & $5.00(1.55)$ & $1-7$ \\
\hline $\begin{array}{l}\text { provide practitioners/you clear instructions about how much PA should be } \\
\text { done in one session? }\end{array}$ & 43 & $4.12(1.59)$ & $1-7$ & 16 & $5.88(1.15)$ & $3-7$ \\
\hline
\end{tabular}

Abbreviations: $\mathrm{M}$, mean; PA, physical activity; $\mathrm{SCl}$, spinal cord injury.

Most items were rated on a seven-point scale with the following anchors: $1=$ definitely no; $7=$ definitely yes. Items marked with an asterisk $\left({ }^{*}\right)$ had the following anchors: $1=$ not all confident; $7=$ completely confident.

Table 3 Guideline evaluation and feedback from health care practitioners and people with SCI

\begin{tabular}{|c|c|}
\hline Suggestions for improvement & Response to feedback \\
\hline \multicolumn{2}{|l|}{ More detail needed regarding } \\
\hline Intensity definitions & Greater detail is given in the 'how much?' column \\
\hline Intensity of activities (aerobic and strength) & $\begin{array}{l}\text { Aerobic intensity definition is based on validated descriptions } \\
\text { of moderate intensity activity for people with } \mathrm{SCl}^{30}\end{array}$ \\
\hline Amount of activity per session & The effort needed is clearly described based on established definitions ${ }^{30}$ \\
\hline How to measure effort & Amount of activity per session is clarified in the 'how much' row \\
\hline Types of activities & $\begin{array}{l}\text { Additional information on activities will be provided in the accompanying } \\
\text { physical activity guide }\end{array}$ \\
\hline \multicolumn{2}{|l|}{ Review } \\
\hline Recommendations about number of repetitions & Guideline has been clarified \\
\hline Aerobic duration & Guideline has been clarified \\
\hline Amount of time of physical activity per week & Time is clearly stated \\
\hline \multicolumn{2}{|l|}{ Add information regarding } \\
\hline Precautions/contraindications/safety & Included in the preamble \\
\hline Sports (aerobic and strength intensity of different sports) & All of this information will be included in the accompanying physical activity \\
\hline Concrete examples of activities and workout plans/routines & guide (under development) \\
\hline Obstacles to physical activity & \\
\hline Benefits of PA & \\
\hline
\end{tabular}

Abbreviations: $\mathrm{PA}$, physical activity; $\mathrm{SCl}$, spinal cord injury.

description of the systematic review process and evaluation of the evidence is described in Hicks et al. ${ }^{16}$

Consensus panel deliberations: Panel members convened for a 2-day meeting in December 2009 in Toronto, Ontario, Canada. The meeting was chaired by a physician-researcher (JS) with expertise in the AGREE II process and the development of clinical practice guidelines. Tables summarizing the evidence ${ }^{16}$ were circulated to members before the meeting. To start the meeting, the Chair led a discussion, followed by straw votes, to determine whether comprehensive guidelines could be developed based on the existing evidence.

The panel noted only 13 studies of acute patients $(<12$ months post injury) and most were of lower methodological quality (that is, pre-post or case study designs). On the basis 
of the quantity and quality of the evidence, and, consistent with the conclusions drawn in the systematic review, ${ }^{16}$ the panel voted unanimously that there was insufficient evidence to develop guidelines to improve fitness among people with acute SCI.

There were 69 studies involving men and women with chronic SCI. Sample sizes ranged from 1 to 133 . There was tremendous heterogeneity across studies in terms of the age of the participants, time since injury and injury level. Of studies that reported the injury level of the participant, the highest injury included was C3 and the lowest was L5. Some studies focused only on people with paraplegia, some only on people with tetraplegia, and others included both paraplegia and tetraplegia. The panel voted unanimously that there was sufficient evidence to develop guidelines to improve muscle strength and physical capacity in the chronic SCI population, across both paraplegic and tetraplegic subgroups. This decision was based on the existence of high-quality evidence (that is, from randomized and nonrandomized controlled trials (RCTs)) along with considerable and consistent lower quality evidence (that is, pre-post and case study designs) that aerobic and resistance exercise training increase physical capacity and muscle strength in people with chronic SCI. ${ }^{16}$

The panel then discussed the evidence regarding the two other fitness outcomes addressed in the systematic review: body composition and functional performance. The systematic review yielded no evidence that exercise can decrease body weight and mixed evidence regarding the effects of exercise on muscle and fat mass. ${ }^{16}$ Given the absence of consistent evidence that exercise can improve these components of body composition, coupled with the overall low-quality of the evidence (all but one of the 24 reviewed papers used a pre-post or case study design), the panel agreed that guidelines could not be developed to improve body composition. Regarding functional performance, the systematic review addressed the effects of exercise on wheelchair propulsion, standing and walking. The panel acknowledged the consistent evidence that exercise can improve wheelchair propulsion but noted that all of these studies were of lower quality (that is, pre-post designs). Similarly, the panel noted all but one study of the effects of exercise on standing and walking used a pre-post or case study design and these studies produced mixed findings. Because there was no clear indication from higher quality evidence that exercise can improve functional performance, the panel agreed that guidelines could not be developed at this time to improve functional performance.

Guidelines to improve physical capacity and muscle strength were then formulated through a directed and iterative discussion aimed at identifying the minimum frequency, intensity and duration required to produce improvements in physical capacity and muscular strength. When weighing the evidence garnered from the studies of people with chronic SCI, RCTs were given priority. Pre-post studies with a higher quality score were more heavily weighted than those with a lower score. The expertise of panel clinicians and consumers was integrated with the systematic review ${ }^{16}$ evidence to derive the draft guidelines.
For both the aerobic exercise and strength-training guidelines, the recommended minimum frequency is 2 days per week. Although most of the reviewed studies used 3 days per week training protocols, high-quality RCT evidence showed the effectiveness of 2 days per week exercise for improving physical capacity and muscular strength over 9 months. ${ }^{3}$ Furthermore, on the basis of clinical and practical expertise, the panel felt that exercise 2 days per week was more realistic and sustainable for people with SCI than exercise $\geqslant 3$ days per week.

Regarding aerobic training intensity, the guideline stipulates moderate-heavy intensity activity. This recommendation is based on high-quality RCT evidence ${ }^{3}$ backed by consistent pre-post study evidence ${ }^{17-19}$ that moderateheavy intensity aerobic training is effective for improving physical capacity. For strength-training intensity, the guideline stipulates an intensity equivalent to $70-80 \%$ of 1 repetition maximum (that is, three sets of 8-10 repetitions of each exercise for each major muscle group). Although the systematic review showed that lower intensities were also effective, the lower intensity training protocols were performed $\geqslant 3$ days per week. Given the panel's recommendation of exercise 2 days per week, the panel felt that a higher intensity guideline was required to achieve benefits.

With regard to aerobic training duration, the guideline indicates $20 \mathrm{~min}$ as a minimum, or starting point. This recommendation was based on high-quality RCT evidence, ${ }^{3}$ coupled with pre-post study evidence $e^{20-22}$ that bouts of aerobic activity $\geqslant 20 \mathrm{~min}$ led to significant increases in physical capacity. The panel acknowledged that most studies used aerobic training $\geqslant 30 \mathrm{~min}$; however, for people with SCI who are just starting an exercise program, 20 min bouts are a more realistic target that yield fitness benefits.

The panel also discussed types of aerobic and strengthtraining activities that confer fitness benefits. As noted in the systematic review, ${ }^{16}$ evidence from randomized and nonRCTs and pre-post studies showed the effectiveness of functional electrical stimulation-assisted exercise, traditional resistance training, arm ergometry and combined aerobic/ resistance exercise for improving both physical capacity and strength. In addition, the panel noted some lower quality studies showed effectiveness for other activities such as treadmill walking, ${ }^{23}$ swimming $^{24}$ and wheelchair ergometry; ${ }^{25}$ panelists with extensive practical expertise also endorsed these activities for improving fitness. Because the panel felt it was important to provide as many activity options as possible, all of these activities were included in a clarification message indicating how one could achieve the guidelines.

In a subsequent discussion, the panel addressed the potential health benefits and risks associated with performing the PA guidelines. In general, there is good evidence that PA confers various health benefits to people with SCI, such as reduced risk for cardiovascular disease and obesity. ${ }^{11,26}$ However, panel members agreed that there is insufficient research evidence to conclude, unequivocally, that the amount of activity recommended in the guidelines is associated with these benefits. Regarding risks associated with exercise, adverse events are seldom reported in the 
PA and SCI literature, but are very low in those studies that do report them. ${ }^{27}$ Muscle pain is the most commonly reported adverse event ${ }^{28}$ and, depending on injury level and severity, risk may increase for exercise-related adverse events including hypotension, arrhythmias and/or musculoskeletal injury. ${ }^{29,30}$ There are insufficient data to draw an evidence-based conclusion regarding the risks associated with performing activity according to the guidelines. However, the panel noted that when proper precautions are taken, the risks are relatively low and that adverse events resulting from the PA guidelines are likely comparable with the variant risks observed in the general population.

Procedures for updating the guidelines were discussed. The panel agreed that the guidelines should be updated annually through a systematic review of the most recent literature and a 1-day consensus panel meeting to discuss the research quality and evidence. Currently, there is no funding for updating.

Clarity and presentation. Two panel members with expertise in health communications (AL) and PA behavior change (KMG) prepared the user-friendly draft of the guidelines for the pilot study (see 'stakeholder involvement'). The language and format of the guidelines were modeled after the Centers for Disease Control's presentation of PA guidelines for adults, ${ }^{28}$ to specify the frequency, intensity, and duration of aerobic and resistance training needed to obtain fitness benefits. The AGREE II protocol ${ }^{13-15}$ indicates that other options for managing a condition be included with the guidelines. Given that PA is the only way to improve fitness, no other options are provided.

On the basis of the feedback of the stakeholders (see Tables 2 and 3), the language of the draft guidelines was modified and some clarification messages were added. For enhanced clarity, and to provide context for the use of the guidelines, a preamble was also added, modeled after the preamble for Canada's PA guidelines. Separate preambles were written for consumers and health care practitioners. The final version with the consumer preamble is presented in Figure 1. The health care practitioner's preamble and French and Chinese language versions are available at http:// www.sciactioncanada.ca.

The AGREE II protocol ${ }^{13-15}$ indicates that there be supporting tools to help with the application of the guidelines. The development and pilot testing of an accompanying PA guide began in January 2010 and is scheduled for release in late 2011. This resource will be a primary mechanism for
PHYSICAL ACTIVITY GUIDELINES

for Adults with Spinal Cord Injury

\section{PREAMBLE}

These guidelines are appropriate for all healthy adults with chronic spinal cord injury, traumatic or non-traumatic, including tetraplegia and paraplegia, irrespective of gender, race, ethnicity or socio-economic status. Adults are encouraged to participate in a variety of physical activities that are enjoyable and safe.

You should try to be active throughout the day, and in a variety of ways, in addition to your usual activities of daily living.

If you are newly injured, are pregnant, prone to autonomic dysreflexia, or have other medical conditions, you should talk to your health professional to find out what types and amount of physical activity are right for you. A health professional might include a doctor, a physiotherapist, or a qualified exercise professional.

As a safe progression towards meeting the guidelines it is appropriate to start with smaller amounts of physical activity and gradually increase how long, how often, and how hard you engage in physical activity.

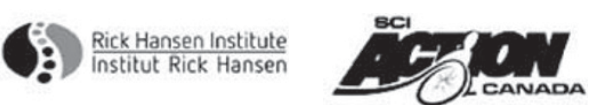

www.sciactioncanada.ca/guidelines

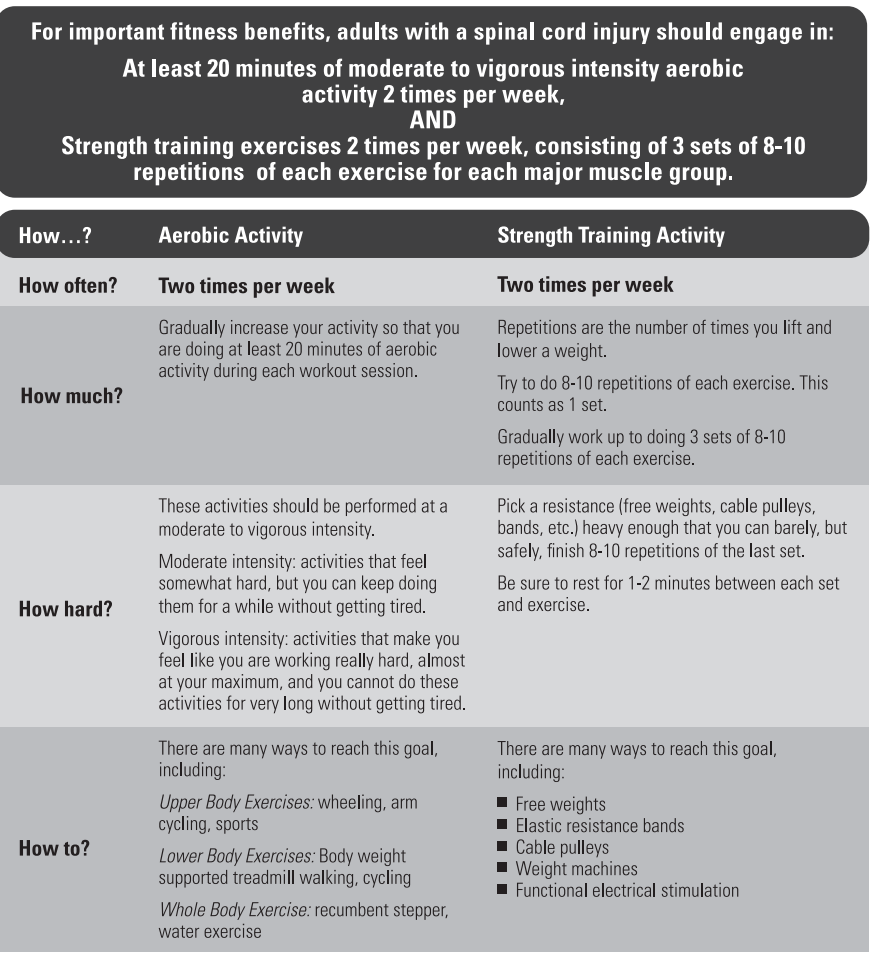

Figure 1 Physical Activity Guidelines and Preamble for Consumers. (A French language and Chinese language version is available from the first author). 
communicating the guidelines and providing strategies to help achieve the guidelines. The consensus panel recommended additional dissemination strategies including publication in scientific journals, presentations at scientific meetings, and creation and inclusion of a SCI-specific module in fitness professional certification courses.

Applicability. In the AGREE II protocol, two aspects of applicability reflect whether the potential organizational barriers and cost implications of applying the recommendations have been considered. The panel discussed organizational barriers such as lack of staff/practitioner knowledge about the benefits of PA for people with SCI, lack of confidence in prescribing PA, resistance to change in current practices and limited funding to provide PA programs in communities for people with SCI. The panel also discussed costs to individuals who want to increase their activity to achieve the guidelines, such as financial costs associated with program membership, attendant care and transportation. Panel members have written about and discussed these barriers and costs in various publications. ${ }^{8,27}$ The AGREE II protocol also recommends addressing potential facilitators for the implementation of new guidelines. Several suggestions were discussed: updating building standards to accommodate access for people using wheelchairs, increasing the presence of accessible training equipment in community fitness facilities and providing personalized resources such as Get in Motion (a free PA counseling service for Canadians with SCI; http://www.sciactioncanada.ca).

The third AGREE II aspect of applicability reflects whether the guidelines have review criteria for monitoring guideline adherence. Adherence will be determined through population-level activity monitoring to assess whether people are physically active at the levels and frequencies indicated in the guidelines. This approach parallels monitoring that takes place in the general Canadian population. ${ }^{28,31}$ The current plan is to monitor guideline adherence annually in a subset of the Canadian SCI population $(n \sim 300)$ through a telephone-administered PA assessment tool (the Physical Activity Recall Assessment-SCI). ${ }^{32}$

Editorial independence. The Rick Hansen Institute is the primary funder of the guideline development project. The views of the Institute had no influence on the final guideline whatsoever. No guideline development group members declared a conflict of interest.

\section{Discussion}

To our knowledge, this is the first attempt to develop SCIspecific PA guidelines using the rigorous, internationally accepted, consensus approach outlined in AGREE II. The expert panel anticipates that the guidelines will have important implications for consumers, clinicians and policy makers by providing a basis for the promotion of PA for people with SCI. The guidelines will also provide a foundation for future PA research, and a benchmark to facilitate PA monitoring and surveillance.
From a practical perspective, the release of the guidelines could result in service providers altering their approach to prescribing and providing exercise to people with SCI. For example, possessing information on how to prescribe PA to people with SCI, exercise programmers and community fitness centers may now be more inclined to provide PA programs to people with disabilities. Similarly, the guidelines and accompanying guide (in development) will remove existing informational barriers that have dissuaded health care practitioners from recommending PA.

Evidence-based guidelines could also provide a foundation for building a case for universal insurance coverage of PA expenses for people with SCI. Application of the guidelines in this context requires strategic dissemination and supporting documentation to key policy and decision makers. The information must also be presented in a way that prohibits the guidelines from being used to scale-back insurance plans that provide coverage for more than the recommended amount of activity.

From a research perspective, the guidelines provide a standardized exercise prescription for use in exercise intervention studies and a benchmark against which to gauge the effects of higher and lower volumes of activity. The SCI and PA literature is marred by inconsistent and varied exercise protocols, rendering it difficult to make between-study comparisons about the effects of exercise. To begin addressing this limitation, researchers are encouraged to use the guidelines when developing their exercise protocols. Furthermore, these guidelines provide the framework to move ahead with important research questions related to determining the secondary health benefits (for example, chronic disease risk) of PA for the SCI population.

\section{Strengths and weaknesses}

High-quality guidelines are developed using systematic methods that combine the social engagement of a multidisciplinary team of stakeholders with rigorous methodology. $^{33}$ Our consensus panel represented experts in the AGREE II process, people experienced in clinical practice guideline development, content experts, SCI consumers and other end users. Although the recommendations of the panel were underpinned by a rigorous systematic review, by far the greatest limitation of our work relates to the current state of the evidence. Compared with the non-disabled population, there are significantly fewer studies of PA involving people with SCI, and many of these studies have not used rigorous methodological designs. After identifying over 160 potentially eligible papers, our systematic review process included only 69 articles that studied PA in people with chronic SCI and 13 with acute SCI. Consequently, there was insufficient evidence to generate guidelines for acute SCI. For the chronic SCI population, there was insufficient evidence to generate guidelines for improving body composition and physical functioning. Furthermore, although the link between fitness and improved health (through risk factor modification) may appear intuitive, the consensus panel felt that there was insufficient evidence to justify PA recommendations related to reducing disease risk. 
Although our development of PA guidelines to improve muscular strength and cardiovascular endurance is an important step forwards, future research is needed to firmly establish the link between improved fitness and secondary disease risk in the SCI population.

\section{Next steps}

Over the coming months, we will work to disseminate the guidelines through key community-based organizations that provide PA information and services to people with SCI and other disabilities. Members of the guideline team are also developing a PA guide with SCI-specific information to assist people in starting and maintaining a PA regimen with the goal of attaining the guideline-specified levels of activity. To assess the impact of the guidelines on PA participation, there is potential to monitor consumer adherence to the guidelines by using a community-based exercise program for people with SCI. The guidelines can be disseminated to program members and researchers can subsequently monitor attendance, duration of exercise bouts, and type of activity undertaken. Comparisons can be made with archival data to determine whether the guidelines have influenced patterns of program participation and exercise adherence. In addition, data from an ongoing cohort study that monitors changes in PA levels among people with SCI living in two Canadian provinces can be used to determine the impact of the guideline release on PA.

In conclusion, our team has undertaken the most rigorous process to date for the development of PA guidelines for people with SCI. We encourage researchers, clinicians and, of course, people with SCI to adopt these guidelines. We also encourage the use of this process to develop PA guidelines for people with other types of disabilities.

\section{Conflict of interest}

The authors declare no conflict of interest.

\section{Acknowledgements}

We gratefully acknowledge Dr Luc Noreau who provided the initial impetus for this project. Development of the guidelines and preparation of the manuscript were financially supported by the Rick Hansen Institute; a CommunityUniversity Research Alliance Grant from the Social Sciences and Humanities Research Council of Canada; a Canadian Institutes of Health Research New Investigator Award (awarded to KMG); an Ontario Neurotrauma Foundation Mentor-Trainee Award (awarded to KMG and AL). We extend our appreciation to Adrienne Sinden for her assistance with preparing the manuscript.

\section{References}

1 Garshick E, Kelley A, Cohen SA, Garrison A, Tun CG, Gagnon D et al. A prospective assessment of mortality in chronic spinal cord injury. Spinal Cord 2005; 43: 408-416.

2 Noreau L, Shephard RJ. Spinal cord injury, exercise and quality of life. Sports Med 1995; 20: 226-250.
3 Hicks AL, Martin KA, Ditor DS, Latimer AE, Craven C, Bugaresti J et al. Long-term exercise training in persons with spinal cord injury: effects on strength, arm ergometry performance and psychological well-being. Spinal Cord 2003; 41: 34-43.

4 Hicks AL, Martin Ginis KA. Treadmill training after SCI: it's not just about the walking. J Rehabil Res Dev 2008; 45: 241-248.

5 Public Health Agency of Canada 1998 Canada's physical activity guide to healthy active living (online). Available from http:// www.phac-aspc.gc.ca/hp-ps/hl-mvs/pag-gap/index-home-accueileng.php (accessed 09 September 2010).

6 American College of Sports Medicine 2008 Guidelines for healthy adults under age 65 (online). Available from http://www.acsm. org/AM/Template.cfm?Section $=$ Home_Page\&TEMPLATE $=/ \mathrm{CM} /$ HTMLDisplay.cfm\&CONTENTID = 7764 (accessed 23 March 2011).

7 World Health Organization 2010. Global Recommendations on Physical Activity for Health. WHO Press: Geneva, Switzerland.

8 Martin Ginis KA, Hicks AL. Factors to be considered in creating a physical activity guide for Canadians with physical disabilities. Appl Physiol Nutr Metab 2007; 32(Suppl 2F): S150-S164.

9 Tremblay MS, Kho ME, Tricco AC, Duggan M. Process description and evaluation of Canadian Physical Activity Guidelines development. Int J Behav Nutr Phys Act 2010; 7: 42.

10 Myers J, Nieman D (eds). ACSM's Resources for Clinical Exercise Physiology, 2nd edn. Wolters Luwer/Lippincott Williams \& Wilkins: Baltimore, 2010.

11 Jacobs PL, Nash MS. Exercise recommendations for individuals with spinal cord injury. Sports Med 2004; 34: 727-751.

12 Myslinski MJ. Evidence-based exercise prescription for individuals with spinal cord injury. J Neurol Phys Ther 2005; 29: 104-106.

13 Brouwers MC, Kho ME, Browman G, Burgers J, Cluzeau F, Feder G et al. AGREE II: advancing guideline development, reporting and evaluation in healthcare. CMAJ 2010; 182: E839-E842.

14 Brouwers MC, Kho ME, Browman G, Burgers J, Cluzeau F, Feder G et al. AGREE II: advancing guideline development, reporting and evaluation in healthcare. J Clin Epidemiol 2010; 63: 1308-1311.

15 Brouwers MC, Kho ME, Browman G, Burgers J, Cluzeau F, Feder G et al. AGREE II: advancing guideline development, reporting and evaluation in healthcare. Prev Med 2010; 51: 421-424.

16 Hicks AL, Martin Ginis KA, Pelletier CA, Ditor DS, Foulon B, Wolfe DL. The effects of exercise training on physical capacity, strength, body composition and functional performance among adults with spinal cord injury: a systematic review. Spinal Cord 2011; 49: 1103-1127.

17 Heesterbeek PJC, Berkelmans HWA, Thijssen DHJ, van Kuppevelt HJM, Hopman MTE, Duysens J. Increased physical fitness after 4week training on a new hybrid FES-cycle in persons with spinal cord injury. Technol Disabil 2005; 17: 103-110.

18 Jacobs PL. Effects of resistance and endurance training in persons with paraplegia. Med Sci Sports Exerc 2009; 41: 992-997.

19 Janssen TW, Pringle DD. Effects of modified electrical stimulation-induced leg cycle ergometer training for individuals with spinal cord injury. J Rehabil Res Dev 2008; 45: 819-830.

20 Hooker SP, Wells CL. Effects of low- and moderate-intensity training in spinal cord-injured persons. Med Sci Sports Exerc 1989; 21: 18-22.

21 Nilsson S, Staff PH, Pruett DR. Physical work capacity and the effect of training on subjects with long-standing paraplegia. Scand J Rehab Med 1975; 7: 51-56.

22 Whiting RB, Dreisinger TE, Dalton RB, Londeree BR. Improved physical fitness and work capacity in quadriplegics by wheelchair exercise. J Cardiac Rehab 1983; 3: 251-255.

23 de Carvalho DC, Martins CL, Cardoso SD, Cliquet Jr A. Improvement of metabolic and cardiorespiratory responses through treadmill gait training with neuromuscular electrical stimulation in quadriplegic subjects. Artif Organs 2006; 30: 56-63.

24 Ornstein LJ, Skrinar GS, Carrett GG. Physiological effects of swimming training in physicaly disabled individuals. Med Sci Sports Exerc 1983; 15: 110.

25 Tordi N, Dugue B, Klupzinski D, Rasseneur L, Rouillon JD, Lonsdorfer J. Interval training program on a wheelchair ergometer for paraplegic subjects. Spinal Cord 2001; 39: 532-537.

26 Buchholz AC, Martin Ginis KA, Bray SR, Craven BC, Hicks AL, Latimer $\mathrm{AE}$ et al. Greater daily leisure time physical activity is 
associated with lower chronic disease risk in adults with spinal cord injury. Appl Physiol Nutr Metab 2009; 34: 640-647.

27 The Spinal Cord Injury Rehabilitation Evidence (SCIRE) 2010 (online) Available from http://www.scireproject.com/ (accessed 09 September 2010).

28 Centers for Disease Control and Prevention 2010. Physical activity for everyone: how much physical activity do adults need? (online). Available at http://www.cdc.gov/physicalactivity/every one/guidelines/adults.html (accessed 9 September 2010).

29 Krassioukov A, Eng JJ, Warburton DE, Teasell R, Spinal cord injury rehabilitation evidence research team. A systematic review of the management of orthostatic hypotension after spinal cord injury. Arch Phys Med Rehabil 2009; 90: 876-885.
30 Krassioukov A, Warburton DE, Teasell R, Eng JJ, Spinal Cord Injury Rehabilitation Evidence Research Team. A systematic review of the management of autonomic dysreflexia after spinal cord injury. Arch Phys Med Rehabil 2009; 90: 682-695.

31 Canada Fitness and Lifestyle Research Institute (CFLRI). Physical activity levels across Canada. (online). Available at http:// www.cflri.ca/eng/levels/index.php (accessed 16 September 2010).

32 Martin Ginis KA, Latimer AE, Hicks AL, Craven BC. Development and evaluation of an activity measure for people with spinal cord injury. Med Sci Sports Exerc 2005; 37: 1099-1111.

33 Brouwers M, Stacey D, O'Connor A. Knowledge creation: synthesis, tools and products. CMAJ 2010; 182: E68-E72. 